\title{
A Malaria Model with Two Delays
}

\author{
Hui Wan ${ }^{1}$ and Jing-an Cui ${ }^{2}$ \\ ${ }^{1}$ Jiangsu Key Laboratory for NSLSCS, School of Mathematical Sciences, Nanjing Normal University, Nanjing 210046, China \\ ${ }^{2}$ School of Sciences, Beijing University of Civil Engineering and Architecture, Beijing 100044, China \\ Correspondence should be addressed to Jing-an Cui; cuijingan@bucea.edu.cn
}

Received 5 November 2012; Accepted 25 January 2013

Academic Editor: Xiang Ping Yan

Copyright (C) 2013 H. Wan and J.-a. Cui. This is an open access article distributed under the Creative Commons Attribution License, which permits unrestricted use, distribution, and reproduction in any medium, provided the original work is properly cited.

\begin{abstract}
A transmission model of malaria with two delays is formulated. We calculate the basic reproduction number $R_{0}$ for the model. It is shown that the basic reproduction number is a decreasing function of two time delays. The existence of the equilibria is studied. Our results suggest that the model undergoes a backward bifurcation, which implies that bringing the basic reproduction number below 1 is not enough to eradicate malaria.
\end{abstract}

\section{Introduction}

Malaria is a mosquito-borne disease which is due to the four species of the genus Plasmodium, namely, Plasmodium falciparum, Plasmodium vivax, Plasmodium malariae, and Plasmodium ovale. These parasites are transmitted to the human host through a bite by an infected female anopheles mosquito.

Sporozoites are injected into a human host, which are carried through the blood to the liver within $30 \mathrm{~min}$ [1]. They invade hepatocytes and undergo a process of asexual replication (exoerythrocytic schizogony) to give rise to 10-40 thousand merozoites per sporozoite. Up to this point, the infection is nonpathogenic and clinically silent. After about 7-9 days, the liver schizonts rupture to release the merozoites into the blood and clinical symptoms, such as fever, pain, chills, and sweats, may develop. Each merozoite invades an erythrocyte and divides to form an erythrocytic schizont containing about 16 daughter merozoites [2]. These merozoites either reinfect fresh erythrocytes, giving rise to cyclical blood-stage infection with a periodicity of $48-72 \mathrm{~h}$, depending on the Plasmodium species, or differentiate into sexual transmission stages called gametocytes. When a second mosquito bites the infected human, the gametocytes are ingested, giving rise to extracellular gametes. In the mosquito midgut, the gametes fuse to form a motile zygote (ookinete), which penetrates the mid-gut wall and forms an oocyst, within which meiosis takes place and haploid sporozoites develop. These sporozoites migrate to the salivary glands. The incubation period within the mosquito may last $8-22$ days [3]. The variation in the length of time is due to the environmental temperature. For $P$. falciparum, the average time is 12 days $[4,5]$. Malaria can also be transmitted through blood transfusion, organ transplantation and transplacental malaria (i.e., congenital malaria) can also be significant in populations which are partially immune to malaria.

The application of mathematics to study of infectious disease appear to have been initiated by Berniulli, 1760. Presently, a lot of mathematical models have been proposed to evaluate and compare control procedures and preventive strategies, and to investigate the relative effects of various sociological, biological, and environmental factors on the spread of diseases ([6-14], etc.). These models have played a very important role in the history and development of epidemiology.

The transmission process involves considerable time delay both in human and in mosquitoes due to the incubation periods of the several forms of the parasites [9]. Several works have been done to consider the effect of delay. Taking account of the incubation period in vector (mosquito), Takeuchi et al. [10] considered a differential-delay model for vectorborne diseases and get the global stability of the endemic equilibrium under appropriate conditions. While Wei et al. [15] proposed a similar model of vector-borne disease which has direct mode of transmission in addition to the vectormediated transmission and proved that the introduction of 
a time delay in the host-to-vector transmission term can destabilize the system and periodic solutions can arise through Hopf bifurcation. In [9], Ruan et al. proposed a delayed RossMacdonald model with delays both in human and in mosquitoes. The effect of time delays on the basic reproduction number and the dynamics of the transmission was studied.

Both the models in $[10,15]$ adopted mass action formulation, where the contact rate depends on the size of the total host population while the model in [9] adopted standard incidence formulation, where the contact rate is assumed to be constant (see [16] for detailed derivation of these incidence functions). The choice of one formulation over the other really depends on the disease being modeled and, in some cases, the need for mathematical (analytical) tractability [17]. A crucial question is which of the two incidence functions is more suitable for modeling infectious disease in general, and malaria in particular? As noted in $[16]$, data $([4,18])$ strongly suggests that standard incidence is better for modeling human diseases than mass action incidence. Consequently, standard incidence rate is adopted in this paper.

\section{Derivation of the Model}

In order to study the impact of incubation periods in both human and mosquitoes on the basic reproduction number and the transmission dynamics of malaria over long periods, we propose a model based on SIRS in human population and SI for the mosquito vector population. Since the mosquito dynamics operates on a much faster time scale than the human dynamics and the turnover of the mosquito population is very high and the total size of vector population is largely exceeding the human total size, the mosquito population can be considered to be at a equilibrium with respect to changes in the human population. Hence, the total number of mosquito population is assumed to be constant.

We formulate an SIRS-SI model for the spread of malaria in the human and mosquito population with the total population size at time $t$ is given by $N_{h}(t)$ and $N_{v}(t)$, correspondingly. For the human population, the three compartments represent individuals who are susceptible, infectious, and partially immune, with sizes at time $t$ denoted by $S_{h}(t), I_{h}(t)$, and $R_{h}(t)$, respectively. The incubation period in a human has duration $\tau_{1} \geq 0$. A susceptible individual infected by a mosquito will not become infectious until $\tau_{1}$ time units later and we assume that no one recovers during this incubation period. At the infectious stage a host may die from the disease, recover into the susceptible class, or may recover with acquired partial immunity. Infectious human hosts are those with infectious gametocytes in the blood stream. Partially immune hosts still have protective antibodies and other immune effectors at low levels. $\Lambda_{h}>0$ is the human input (birth) rate. $\mu_{h}>0$ and $\alpha_{h} \geq 0$ are the natural and diseaseinduced death rates, respectively. $\gamma_{h}>0$ is the rate at which human hosts acquire immunity. $\theta \geq 0$ is the per capita rate of recovery into the susceptible class from being infectious. $\rho \geq 0$ is the per capita rate of loss of immunity in human hosts. $\beta_{h}>0$ is the proportion of bites on man that produce an infection. For the mosquito population, the two compartments represent susceptible and infectious mosquitoes, with sizes at time $t$ denoted by $S_{v}(t)$ and $I_{v}(t)$, respectively. The vector component of the model does not include immune class as mosquitoes never recover from infection that is, their infective period ends with their death due to their relatively short life cycle. Thus the immune class in the mosquito population is negligible and death occurs equally in all groups. Our model also excludes the immature mosquitoes since they do not participate in the infection cycle and are, thus, in the waiting period, which limits the vector population growth. We assume that the mosquito population is constant and equal to $N_{v}$, with birth and death rate constants equal to $\mu_{v}$. $\beta_{v}$ is the probability that a mosquito becomes infectious when it bites an infectious human. The biting rate $b$ of mosquitoes is the average number of bites per mosquito per day. This rate depends on a number of factors, in particular, climatic ones, but for simplicity in this paper, we assume $b$ constant. The incubation interval in the mosquito has duration $\tau_{2} \geq 0$.

Considering the assumption made above, the interaction between human hosts and the mosquito vector population with standard incidence rate is described as shown below:

$$
\begin{aligned}
\frac{d S_{h}}{d t}= & \Lambda_{h}-\frac{b \beta_{h}}{N_{h}\left(t-\tau_{1}\right)} I_{v}\left(t-\tau_{1}\right) S_{h}\left(t-\tau_{1}\right) e^{-\mu_{h} \tau_{1}}-\mu_{h} S_{h} \\
& +\theta I_{h}+\rho R_{h}, \\
\frac{d I_{h}}{d t}= & \frac{b \beta_{h}}{N_{h}\left(t-\tau_{1}\right)} I_{v}\left(t-\tau_{1}\right) S_{h}\left(t-\tau_{1}\right) e^{-\mu_{h} \tau_{1}} \\
& -\left(\gamma_{h}+\mu_{h}+\alpha_{h}+\theta\right) I_{h}, \\
\frac{d R_{h}}{d t}= & \gamma_{h} I_{h}-\mu_{h} R_{h}-\rho R_{h}, \\
\frac{d S_{v}}{d t}= & \mu_{v} S_{v}+\mu_{v} I_{v} \\
& -\frac{b \beta_{v}}{N_{h}\left(t-\tau_{2}\right)} I_{h}\left(t-\tau_{2}\right) S_{v}\left(t-\tau_{2}\right) e^{-\mu_{v} \tau_{2}}-\mu_{v} S_{v}, \\
\frac{d I_{v}}{d t}= & \frac{b \beta_{v}}{N_{h}\left(t-\tau_{2}\right)} I_{h}\left(t-\tau_{2}\right) S_{v}\left(t-\tau_{2}\right) e^{-\mu_{v} \tau_{2}}-\mu_{v} I_{v}, \\
\frac{d N_{h}}{d t}= & \Lambda \mu_{h}-\mu_{h} N_{h}-\alpha_{h} I_{h},
\end{aligned}
$$

where $S_{h}(t), I_{h}(t)$, and $R_{h}(t)$ denote the number of susceptible, infective, and recovered in the human population, and $S_{v}(t), I_{v}(t)$ denote the number of susceptible and infective in the mosquito vector population. In addition, $S_{h}+I_{h}+R_{h}=N_{h}$ and $S_{v}+I_{v}=N_{v}$.

System (1) satisfies the initial conditions: $S_{h}(\theta)=S_{h 0}(\theta)$, $I_{h}(\theta)=I_{h 0}(\theta), R_{h}(\theta)=R_{h 0}(\theta), S_{v}(\theta)=S_{v 0}(\theta), I_{v}(\theta)=I_{v 0}(\theta)$, $\theta \in[-\tau, 0]$, where $\tau=\max \left\{\tau_{1}, \tau_{2}\right\}$. The nonnegative cone of $R^{6}$ space $S_{h} I_{h} R_{h} S_{v} I_{v} N_{h}$ is positively invariant for system (1) since the vector field on the boundary does not point to the exterior. What is more, since $d N_{h} / d t<0$ for $N_{h}>\Lambda_{h} / \mu_{h}$ 
and $N_{v}$ is constant, all trajectories in the first quadrant enter or stay inside the region

$$
Z_{+}=\left\{S_{h}+I_{h}+R_{h}=N_{h} \leqslant \frac{\Lambda_{h}}{\mu_{h}}, S_{v}+I_{v}=N_{v}\right\} .
$$

The continuity of the right-hand side of (1) implies that unique solutions exist on a maximal interval. Since solutions approach, enter or stay in $D_{+}$, they are eventually bounded and hence exist for $t>0$. Therefore, the initial value problem for system (1) is mathematically well posed and biologically reasonable since all variables remain nonnegative.

In order to reduce the number of parameters and simplify system (1), we normalize the human and mosquito vector population $s_{h}=S_{h} /\left(\Lambda_{h} / \mu_{h}\right), i_{h}=I_{h} /\left(\Lambda_{h} / \mu_{h}\right), r_{h}=R_{h} /\left(\Lambda_{h} /\right.$ $\left.\mu_{h}\right), n_{h}=N_{h} /\left(\Lambda_{h} / \mu_{h}\right), s_{v}=S_{v} / N_{v}, i_{v}=I_{v} / N_{v}$, and $m=$ $N_{v} /\left(\Lambda_{h} / \mu_{h}\right)$.

Since $r_{h}=n_{h}-s_{h}-i_{h}$ and $s_{v}=1-i_{v}$, the dynamics of system (1) is qualitatively equivalent to the dynamics of system given by

$$
\begin{aligned}
\frac{d s_{h}}{d t}= & \mu_{h}-\frac{b \beta_{h} m}{n_{h}\left(t-\tau_{1}\right)} i_{v}\left(t-\tau_{1}\right) s_{h}\left(t-\tau_{1}\right) e^{-\mu_{h} \tau_{1}} \\
& -\mu_{h} s_{h}+\theta i_{h}+\rho\left(n_{h}-s_{h}-i_{h}\right), \\
\frac{d i_{h}}{d t}= & \frac{b \beta_{h} m}{n_{h}\left(t-\tau_{1}\right)} i_{v}\left(t-\tau_{1}\right) s_{h}\left(t-\tau_{1}\right) e^{-\mu_{h} \tau_{1}} \\
& -\left(\gamma_{h}+\mu_{h}+\alpha_{h}+\theta\right) i_{h}, \\
\frac{d i_{v}}{d t}= & \frac{b \beta_{v}}{n_{h}\left(t-\tau_{2}\right)} i_{h}\left(t-\tau_{2}\right)\left(1-i_{v}\left(t-\tau_{2}\right)\right) e^{-\mu_{v} \tau_{2}}-\mu_{v} i_{v}, \\
\frac{d n_{h}}{d t}= & \mu_{h}-\mu_{h} n_{h}-\alpha_{h} i_{h},
\end{aligned}
$$

and all trajectories in the nonnegative cone $R_{+}^{4}$ enter or stay inside the region $D=\left\{0 \leqslant s_{h}, 0 \leqslant i_{h}, s_{h}+i_{h} \leqslant n_{h} \leqslant 1,0 \leqslant\right.$ $\left.i_{v} \leqslant 1\right\}$.

Define the basic reproduction number by

$$
R_{0}=\frac{m b^{2} \beta_{h} \beta_{v} e^{-\mu_{h} \tau_{1}} e^{-\mu_{v} \tau_{2}}}{\mu_{v}\left(\gamma_{h}+\mu_{h}+\alpha_{h}+\theta\right)} .
$$

It represents the expected number of secondary cases caused by a single infected individual introduced into an otherwise susceptible population of hosts and vectors. Take a primary case of host with a natural death rate of $\mu_{h}$, the average time spent in an infectious state is $1 /\left(\gamma_{h}+\mu_{h}+\alpha_{h}+\theta\right)$. Since the incubation period in mosquitoes has duration $\tau_{2}$, during which period some mosquitoes may die, the average number of mosquito bites received from $m$ susceptible mosquitoes each with a biting rate $b$ gives a total of $b \beta_{v} m e^{-\mu_{v} \tau_{2}} /\left(\gamma_{h}+\right.$ $\left.\mu_{h}+\alpha_{h}+\theta\right)$ mosquitoes successfully infected by the primary human case. Each of these mosquitoes survives for an average time $1 / \mu_{v}$. Because of another incubation period $\tau_{1}$ in human, during which period some individuals may die, totally $b \beta_{h} e^{-\mu_{h} \tau_{1}} / \mu_{v}$ hosts will be infected by a single mosquito.
Therefore, the total number of secondary cases is thus $m b^{2} \beta_{h} \beta_{v} e^{-\mu_{h} \tau_{1}} e^{-\mu_{v} \tau_{2}} / \mu_{v}\left(\gamma_{h}+\mu_{h}+\alpha_{h}+\theta\right)$, which is the basic reproduction number $R_{0}$.

\section{Existence of Equilibria}

Equating the derivatives on the left-hand side to zero and solving the resulting algebraic equations. The points of equilibrium $\widehat{P}\left(\widehat{s}_{h}, \widehat{i}_{h}, \widehat{i}_{v}, \widehat{n}_{h}\right)$ satisfy the following relations:

$$
\begin{gathered}
\widehat{s}_{h}=\frac{\mu_{h}+\rho-H \widehat{i}_{h}}{\mu_{h}+\rho}, \\
\widehat{n}_{h}=\frac{\mu_{h}-\alpha_{h} \widehat{i}_{h}}{\mu_{h}}, \\
\widehat{i}_{v}=\frac{b \beta_{v} \mu_{h} \hat{i}_{h} e^{-\mu_{v} \tau_{2}}}{\left(b \beta_{v} \mu_{h} e^{-\mu_{v} \tau_{2}}-\alpha_{h} \mu_{v}\right) \hat{i}_{h}+\mu_{v} \mu_{h}},
\end{gathered}
$$

where $H=\gamma_{h}+\mu_{h}+\alpha_{h}+\rho+\rho\left(\alpha_{h} / \mu_{h}\right)$. Substituting (5) in the corresponding second equilibrium equation of (3), we obtain that the solutions are $\widehat{i}_{h}=0$, and the zero points of the quadratic polynomial

$$
r\left(i_{h}\right)=a_{1} i_{h}^{2}+a_{2} i_{h}+a_{3}
$$

where

$$
\begin{gathered}
a_{1}=\left(b \beta_{v} \mu_{h} e^{-\mu_{v} \tau_{2}}-\alpha_{h} \mu_{v}\right) \frac{\alpha_{h}}{\mu_{h}}, \\
a_{2}=2 \alpha_{h} \mu_{v}-b \beta_{v} \mu_{h} e^{-\mu_{v} \tau_{2}} \\
\quad-\frac{m b^{2} \beta_{h} \beta_{v} \mu_{h} H}{\left(\mu_{h}+\rho\right)\left(\gamma_{h}+\mu_{h}+\alpha_{h}+\theta\right)} e^{-\mu_{h} \tau_{1}} e^{-\mu_{v} \tau_{2}}, \\
a_{3}=\mu_{h} \mu_{v}\left(R_{0}-1\right), \\
R_{0}=\frac{m b^{2} \beta_{h} \beta_{v} e^{-\mu_{h} \tau_{1}} e^{-\mu_{v} \tau_{2}}}{\mu_{v}\left(\gamma_{h}+\mu_{h}+\alpha_{h}+\theta\right)} .
\end{gathered}
$$

The solution $\hat{i}_{h}=0$ gives the disease-free equilibrium point $P_{0}(1,0,0,1)$. We are looking for nontrivial equilibrium solutions in the interior of $\Omega$. From (5), in order to keep $\widehat{s}_{h}$ and $\widehat{n}_{h}$ positive, $\widehat{i}_{h} \in\left(0,\left(\mu_{h}+\rho\right) / H\right)$ must be satisfied. Evaluating $r\left(i_{h}\right)$ at the end points of the interval, we obtain

$$
\begin{gathered}
r(0)=\mu_{h} \mu_{v}\left(R_{0}-1\right), \\
r\left(\frac{\mu_{h}+\rho}{H}\right)<0 .
\end{gathered}
$$

When $R_{0}>1$, then $r(0)>0$; therefore, there exists a unique root in the interval $\left(0,\left(\mu_{h}+\rho\right) / H\right)$, which implies the existence of a unique equilibrium point $P^{*}\left(\widehat{s}_{h}^{*}, \widehat{i}_{h}^{*}, \widehat{i}_{v}^{*}, \widehat{n}_{h}^{*}\right)$, where $\widehat{i}_{h}^{*}$ satisfies: if $a_{1}=0$,

$$
\widehat{i}_{h}^{*}=\frac{-a_{3}}{a_{2}} .
$$


If $a_{1} \neq 0$,

$$
\widehat{i}_{h}^{*}=\frac{-a_{2}-\sqrt{\Delta}}{2 a_{1}},
$$

where $\Delta=a_{2}^{2}-4 a_{1} a_{3}$.

When $R_{0}=1$, the roots of (6) are 0 and $-a_{2} / a_{1}$. It is easy to see that there exists a unique root in the interval $\left(0,\left(\mu_{h}+\right.\right.$ $\rho) / H$ ) if and only if $a_{2}>0$, which implies the existence of a unique equilibrium point $P^{*}$.

When $R_{0}<1$, then $r(0)<0$. The conditions to have at least one root in the mentioned interval are $a_{1}<0,0<$ $-a_{2} / 2 a_{1}<\left(\mu_{h}+\rho\right) / H$ and $\Delta=a_{2}^{2}-4 a_{1} a_{3} \geqslant 0$. If $\Delta>0$, there exist two roots in the interval mentioned above, which implies the existence of two equilibria $P_{1}\left(\widehat{s}_{h 1}, \widehat{i}_{h 1}, \widehat{i}_{v 1}, \widehat{n}_{h 1}\right)$ and $P_{2}\left(\widehat{s}_{h 2}, \widehat{i}_{h 2}, \widehat{i}_{v 2}, \widehat{n}_{h 2}\right)$, where

$$
\begin{aligned}
& \widehat{i}_{h 1}=\frac{-a_{2}+\sqrt{\Delta}}{2 a_{1}}, \\
& \widehat{i}_{h 2}=\frac{-a_{2}-\sqrt{\Delta}}{2 a_{1}} .
\end{aligned}
$$

If $\Delta=0$, there is a unique root in the interval mentioned above, which implies the existence of unique equilibrium.

Then, we can conclude the above results in the following theorem.

Theorem 1. (1) The disease-free equilibrium $P_{0}(1,0,0,1)$ always exists.

(2) If $R_{0}>1$, there exists a unique positive equilibrium $P^{*}\left(\widehat{s}_{h}^{*}, \widehat{i}_{h}^{*}, \widehat{i}_{v}^{*}, \widehat{n}_{h}^{*}\right)$.

(3) If $R_{0}=1$, then there is a positive equilibrium $P^{*}\left(\widehat{s}_{h}^{*}\right.$, $\left.\widehat{i}_{h}^{*}, \widehat{i}_{v}^{*}, \widehat{n}_{h}^{*}\right)$ when $a_{2}>0$; otherwise, there is no positive equilibrium.

(4) If $R_{0}<1$, then (a) if $a_{1} \geqslant 0$, there is no positive equilibrium; (b) if $a_{1}<0$, the system (3) has two positive equilibria $P_{1}\left(\widehat{s}_{h 1}, \widehat{i}_{h 1}, \widehat{i}_{v 1}, \widehat{n}_{h 1}\right)$ and $P_{2}\left(\widehat{s}_{h 2}, \widehat{i}_{h 2}, \widehat{i}_{v 2}, \widehat{n}_{h 2}\right)$ if and only if $\Delta>0$ and $0<-a_{2} / 2 a_{1}<\left(\mu_{h}+\rho\right) / H$, and these two equilibria merge with each other if and only if $0<-a_{2} / 2 a_{1}<\left(\mu_{h}+\rho\right) / H$ and $\Delta=0$; otherwise, there is no positive equilibrium.

\section{Disease-Free Equilibrium}

It is easy to see the disease-free equilibrium $P_{0}(1,0,0,1)$ always exists. In this section, we study the stability of the disease-free equilibrium $P_{0}$.

Theorem 2. $P_{0}$ is locally stable if $R_{0}<1 . P_{0}$ is a degenerate equilibrium of codimension one and is stable except in one direction if $R_{0}=1 . P_{0}$ is unstable if $R_{0}>1$.

Proof. Linearizing the system (3) at $P_{0}$. The eigenvalues of the Jacobian matrix $J\left(P_{0}\right)$ are $-\mu_{h}-\rho,-\mu_{h}$ and the solutions of the following transcendental equation:

$$
\begin{aligned}
& \left(\lambda+\mu_{v}\right)\left(\gamma_{h}+\mu_{h}+\alpha_{h}+\theta\right) \\
& \quad-m b^{2} \beta_{h} \beta_{v} e^{-\mu_{h} \tau_{1}-\mu_{v} \tau_{2}} e^{-\left(\tau_{1}+\tau_{2}\right) \lambda}=0 .
\end{aligned}
$$

Let

$$
\begin{aligned}
F\left(\lambda, \tau_{1}, \tau_{2}\right)= & \left(\lambda+\mu_{v}\right)\left(\gamma_{h}+\mu_{h}+\alpha_{h}+\theta\right) \\
& -m b^{2} \beta_{h} \beta_{v} e^{-\mu_{h} \tau_{1}-\mu_{v} \tau_{2}} e^{-\left(\tau_{1}+\tau_{2}\right) \lambda}
\end{aligned}
$$

Clearly, $F\left(\lambda, \tau_{1}, \tau_{2}\right)$ is an analytic function. $F\left(0, \tau_{1}, \tau_{2}\right)=$ $\mu_{v}\left(\gamma_{h}+\mu_{h}+\alpha_{h}+\theta\right)\left(1-R_{0}\right)$, and $F(\lambda, 0,0)=\left(\lambda+\mu_{v}\right)\left(\gamma_{h}+\mu_{h}+\right.$ $\left.\alpha_{h}+\theta\right)-m b^{2} \beta_{h} \beta_{v}$. In the following, we discuss the distribution of the solutions of (12) in three cases.

(i) $R_{0}<1$, then $F\left(0, \tau_{1}, \tau_{2}\right)>0$. Since $F_{\lambda}^{\prime}\left(\lambda, \tau_{1}, \tau_{2}\right)>0$ for $\lambda \geq 0, \tau_{1}>0$ and $\tau_{2}>0$, (12) has no zero root and positive real roots for all positive $\tau_{1}$ and $\tau_{2}$. Now we claim that (12) does not have any purely imaginary roots. Suppose that (12) has a pair of purely imaginary roots $\omega i, \omega>0$ for some $\tau_{1}$ and $\tau_{2}$. Then, by calculation, $\omega$ must be a positive real root of

$$
\begin{aligned}
\omega^{4}+ & {\left[\left(\gamma_{h}+\mu_{h}+\alpha_{h}+\theta\right)^{2}+\mu_{v}^{2}\right] \omega^{2}+\left(\gamma_{h}+\mu_{h}+\alpha_{h}+\theta\right)^{2} \mu_{v}^{2} } \\
& -\left(m b^{2} \beta_{h} \beta_{v} e^{-\mu_{h} \tau_{1}-\mu_{v} \tau_{2}}\right)^{2}=0 .
\end{aligned}
$$

However, it is easy to see that (14) does not have nonnegative real roots when $R_{0}<1$. Hence, (12) does not have any purely imaginary roots.

On the other hand, one can easily get that the roots of $F(\lambda, 0,0)=0$ all have negative real parts when $R_{0}<1$. By the implicit function theorem and the continuity of $F\left(\lambda, \tau_{1}, \tau_{2}\right)$, we know that all roots of (12) have negative real parts for positive $\tau_{1}$ and $\tau_{2}$, which implies that $P_{0}$ is stable.

(ii) $R_{0}=1$, then $F\left(0, \tau_{1}, \tau_{2}\right)=0$. Since $F_{\lambda}^{\prime}\left(\lambda, \tau_{1}, \tau_{2}\right)>0$ for $\lambda \geq 0, \tau_{1}>0$ and $\tau_{2}>0$, (12) has a simple zero root and no positive root for all positive $\tau_{1}$ and $\tau_{2}$. Using a similar argument as in (i), we can obtain that except a zero root, all roots of (12) have negative real parts for positive $\tau_{1}$ and $\tau_{2}$. Thus, $P_{0}$ is a degenerate equilibrium of codimension one and is stable except in one direction.

(iii) $R_{0}>1$, then $F\left(0, \tau_{1}, \tau_{2}\right)<0$. Since $\lim _{\lambda \rightarrow \infty} F(\lambda$, $\left.\tau_{1}, \tau_{2}\right)=\infty$ and $F_{\lambda}^{\prime}\left(\lambda, \tau_{1}, \tau_{2}\right)>0$ for $\lambda \geq 0, \tau_{1}>0$ and $\tau_{2}>0$, (12) has a unique positive real root for all positive $\tau_{1}$ and $\tau_{2}$ and $P_{0}$ is unstable.

Theorem 3. If $R_{0} \leq 1$, the disease-free equilibrium $P_{0}$ is globally asymptotically stable when $\alpha=0$.

Proof. We denote by $x_{t}$ the translation of the solution of the system (3), that is, $x_{t}=\left(s_{h}(t+\theta), i_{h}(t+\theta), i_{v}(t+\theta)\right)$, $n_{h}(t+\theta)$ where $\theta \in[-\tau, 0], \tau=\max \left\{\tau_{1}, \tau_{2}\right\}$. In order to prove 
the globally stability of $P_{0}$, we define a Lyapunov function:

$$
\begin{aligned}
L\left(x_{t}\right)= & e^{\mu_{h} \tau_{1}} i_{h}+\frac{m b \beta_{h}}{\mu_{v}} e^{\mu_{v} \tau_{2}} i_{v}(t) \\
& +m b \beta_{h} \int_{t-\tau_{1}}^{t} i_{v}(t) \frac{s_{h}(t)}{n_{h}(t)} d t \\
& +\frac{m b^{2} \beta_{h} \beta_{v}}{\mu_{v}} \int_{t-\tau_{2}}^{t} i_{h}(t) \frac{1-i_{v}(t)}{n_{h}(t)} d t .
\end{aligned}
$$

The derivative of $L$ along the solutions of (3) is given by

$$
\begin{aligned}
\frac{d L\left(x_{t}\right)}{d t}= & -\left(\mu_{h}+\gamma_{h}+\theta\right) e^{\mu_{h} \tau_{1}} i_{h}(t)\left(1-R_{0} e^{-\mu_{h} \tau_{1}} \frac{1-i_{v}(t)}{n_{h}(t)}\right) \\
& -m b \beta_{h} e^{\mu_{v} \tau_{2}} i_{v}(t)\left(1-\frac{s_{h}(t)}{n_{h}(t)} e^{-\mu_{v} \tau_{2}}\right) .
\end{aligned}
$$

Since $\lim _{t \rightarrow \infty} n_{h}=1$ and $R_{0} \leq 1$, we can easily get that $d F / d t \leq 0$ and the set $\left\{P_{0}\right\}$ is the largest invariant set within the set where $d L\left(x_{t}\right) / d t=0$. By LaSalle-Lyapunov Theorem, all trajectories approach $P_{0}$ as $t \rightarrow \infty$.

Usually, the disease can be controlled if the basic reproduction number is smaller than one. Nevertheless, Theorem 1 indicates the possibility of backward bifurcation (where the locally asymptotically stable DFE coexists with endemic equilibrium when $R_{0}<1$ ) when $a_{1}<0$ for the model (3) and it becomes impossible to control the disease by just reducing the basic reproduction number below one. Therefore a further threshold condition beyond the basic reproduction is essential for the control of the spread of malaria.

To check for this, the discriminant $\Delta$ is set to zero and solved for the critical value of $R_{0}$, denoted by $\widehat{R}_{0}$, given by

$$
\widehat{R}_{0}=1+\frac{a_{2}^{2}}{4 a_{1} \mu_{h} \mu_{v}} .
$$

Thus, backward bifurcation would occur for values of $R_{0}$ such that $\widehat{R}_{0}<R_{0}<1$. This is illustrated by simulating the model with the following set of parameter values (it should be stated that these parameters are chosen for illustrative purpose only, and may not necessarily be realistic epidemiologically).

Let $\mu_{h}=1 / 22000, \mu_{v}=1 / 14, \beta_{h}=0.3, \beta_{v}=0.95, m=$ $0.5, \alpha_{h}=0.05, \gamma_{h}=0.26, b=0.5, \rho=0.01, \theta=0.08, \tau_{1}=15$. With this set of parameters, $\widehat{R}_{0}=0.093970<1$. When $\tau_{2}=$ $10, R_{0}=0.62555<1$, so that $\widehat{R}_{0}<R_{0}<1$. There are two positive roots for (6), which are $\widehat{i}_{h 1}=.3501527770 e-3$ and $\widehat{i}_{h 2}=.8864197396 e-3$.

Thus, the following result is established.

Lemma 4. The model (3) undergoes backward bifurcation when $a_{1}<0$ and $0<-a_{2} / 2 a_{1}<\left(\mu_{h}+\rho\right) / H$ holds and $\widehat{R}_{0}<R_{0}<1$.

Epidemiologically, because of the existence of backward bifurcation, whether malaria will prevail or not depends on the initial states. $R_{0}<1$ is no longer sufficient for disease elimination. In order to eradicate the disease, we have a subthreshold number $\widehat{R}_{0}$ and it is now necessary to reduce $R_{0}$ to a value less than $\widehat{R}_{0}$.

The existence of the subthreshold condition $\widehat{R}_{0}$ also has some implications. In some areas where malaria dies out $\left(R_{0}<\widehat{R}_{0}\right)$, it is possible for the disease to reestablish itself in the population because of a small change in environmental or control variables which increase the basic reproduction number above. On the other hand, in some areas with endemic malaria, it may be possible to eradicate the disease with small increasing in control programs such that the basic reproduction number less than $\widehat{R}_{0}$.

From the expression of $R_{0}$, we can see that the basic reproduction number is a decreasing function of both time delays. The time delays have important effect on the basic reproduction number. Especially, since global warming decreases the duration of incubation period and $R_{0}$ increases with the decreasing of $\tau_{1}$ or $\tau_{2}$, global warming will affect the transmission of malaria a lot.

From Theorem 3, if there is no disease-induced death, backward bifurcation will not occur and there is a unique endemic equilibrium when $R_{0}>1$. Therefore, the diseaseinduced death is the cause of the backward bifurcation phenomenon in the model (3).

\section{Endemic Equilibria}

To obtain precise results, we first assume that malaria does not produce significant mortality $(\alpha=0)$. The assumption is not justifiable in all regions where malaria is endemic but it is a useful first approximation. In this part, we will determine the stability of the unique endemic equilibria $P^{*}$ when $R_{0}>1$.

Linearizing the system (3) at $P^{*}$. The eigenvalues of the Jacobian matrix $J\left(P^{*}\right)$ are $-\mu_{h}$ and the solutions of the following transcendental equation:

$$
\lambda^{3}+A_{1} \lambda^{2}+A_{2} \lambda+A_{3}=0
$$

where

$$
\begin{aligned}
A_{1}= & 2 \mu_{h}+\gamma_{h}+\rho+\theta+\mu_{v} \\
& +m b \beta_{h} \hat{i}_{v} e^{-\mu_{h} \tau_{1}} e^{-\lambda \tau_{1}}+b \beta_{v} \widehat{i}_{h} e^{-\mu_{v} \tau_{2}} e^{-\lambda \tau_{2}}, \\
A_{2}= & \left(\mu_{h}+\rho\right)\left(\gamma_{h}+\mu_{h}+\theta\right)+\left(\mu_{h}+\rho\right) \mu_{v} \\
& +\left(\gamma_{h}+\mu_{h}+\theta\right) \mu_{v} \\
& +\left(\mu_{h}+\rho+\gamma_{h}+\mu_{h}+\theta\right) b \beta_{v} \widehat{i}_{h} e^{-\mu_{v} \tau_{2}} e^{-\lambda \tau_{2}} \\
& -\mu_{v}\left(\gamma_{h}+\mu_{h}+\theta\right) e^{-\left(\tau_{1}+\tau_{2}\right) \lambda} \\
& +\left(\gamma_{h}+\mu_{h}+\rho+\mu_{v}+b \beta_{v} \hat{i}_{h} e^{-\mu_{v} \tau_{2}} e^{-\lambda \tau_{2}}\right) \\
& \times m b \beta_{h} \hat{i}_{v} e^{-\mu_{h} \tau_{1}} e^{-\lambda \tau_{1}},
\end{aligned}
$$




$$
\begin{aligned}
A_{3}= & \left(\mu_{h}+\rho\right)\left(\gamma_{h}+\mu_{h}+\theta\right) \mu_{v} \\
& +\left(\mu_{h}+\rho\right)\left(\gamma_{h}+\mu_{h}+\theta\right) b \beta_{v} \widehat{i}_{h} e^{-\mu_{v} \tau_{2}} e^{-\lambda \tau_{2}} \\
& -\left(\mu_{h}+\rho\right)\left(\gamma_{h}+\mu_{h}+\theta\right) \mu_{v} e^{-\left(\tau_{1}+\tau_{2}\right) \lambda} \\
& +\left(\gamma_{h}+\mu_{h}+\rho\right) m b \beta_{h} \widehat{i}_{v} e^{-\mu_{h} \tau_{1}} e^{-\lambda \tau_{1}} \\
& \times\left(\mu_{v}+b \beta_{v} \widehat{i}_{h} e^{-\mu_{v} \tau_{2}} e^{-\lambda \tau_{2}}\right) .
\end{aligned}
$$

For any nonnegative $\tau_{1}$ and $\tau_{2}$, we have the following proposition.

Proposition 5. For the endemic equilibrium $P^{*}\left(\widehat{s}_{h}^{*}, \widehat{i}_{h}^{*}, \widehat{i}_{v}^{*}, \widehat{n}_{h}^{*}\right)$ of the system with characteristic equation (18), one always has

$$
A_{1}>0, \quad A_{2}>0, \quad A_{3}>0, \quad A_{1} A_{2}-A_{3}>0 .
$$

Proof. It is clear that $A_{1}>0, A_{2}>0$ and $A_{3}>0$. For $A_{1} A_{2}-$ $A_{3}$, we have

$$
\begin{aligned}
& A_{1} A_{2}-A_{3}=\left(\gamma_{h}+\mu_{h}+\rho\right) \\
& \times\left\{\left(\mu_{h}+\rho\right)\left(\gamma_{h}+\mu_{h}+\theta\right)\right. \\
& +\left(\mu_{h}+\rho\right) \mu_{v}+\left(\gamma_{h}+\mu_{h}+\theta\right) \mu_{v} \\
& +\left(\mu_{h}+\rho+\gamma_{h}+\mu_{h}+\theta\right) b \beta_{v} \widehat{i}_{h} e^{-\mu_{v} \tau_{2}} e^{-\lambda \tau_{2}} \\
& -\mu_{v}\left(\gamma_{h}+\mu_{h}+\theta\right) e^{-\left(\tau_{1}+\tau_{2}\right) \lambda} \\
& \left.+\left(\gamma_{h}+\mu_{h}+\rho+\mu_{v}\right) m b \beta_{h} \widehat{i}_{v} e^{-\mu_{h} \tau_{1}} e^{-\lambda \tau_{1}}\right\} \\
& +\left(m b \beta_{h} \widehat{i}_{v} e^{-\mu_{h} \tau_{1}} e^{-\lambda \tau_{1}}+\mu_{h}+\theta\right) A_{2} \\
& +\left(\mu_{v}+b \beta_{v} \hat{i}_{h} e^{-\mu_{v} \tau_{2}} e^{-\lambda \tau_{2}}\right) \\
& \times\left\{\left(\mu_{h}+\rho\right) \mu_{v}+\left(\gamma_{h}+\mu_{h}+\theta\right) \mu_{v}\right. \\
& +\left(\mu_{h}+\rho+\gamma_{h}+\mu_{h}+\theta\right) b \beta_{v} \widehat{i}_{h} e^{-\mu_{v} \tau_{2}} e^{-\lambda \tau_{2}} \\
& +\left(\gamma_{h}+\mu_{h}+\rho+\mu_{v}+b \beta_{v} \widehat{i}_{h} e^{-\mu_{v} \tau_{2}} e^{-\lambda \tau_{2}}\right) \\
& \times m b \beta_{h} \widehat{i}_{v} e^{-\mu_{h} \tau_{1}} e^{-\lambda \tau_{1}} \\
& \left.-\mu_{v}\left(\gamma_{h}+\mu_{h}+\theta\right) e^{-\left(\tau_{1}+\tau_{2}\right) \lambda}\right\} \\
& +\left(\mu_{h}+\rho\right)\left(\gamma_{h}+\mu_{h}+\theta\right) \mu_{\nu} e^{-\left(\tau_{1}+\tau_{2}\right) \lambda} \\
& >0 \text {. }
\end{aligned}
$$

Case 1. When $\tau_{1}=\tau_{2}=0$, as a result of Proposition 5 and Hurwitz criterion, all roots of the characteristic equation (18) have negative real parts and the endemic equilibrium $P^{*}$ of (3) is stable when $\tau_{1}=\tau_{2}=0$.

Case 2. When $\tau_{1}>0, \tau_{2}=0$, the characteristic equation (18) becomes

$$
\lambda^{3}+A_{11} \lambda^{2}+A_{21} \lambda+A_{31}=e^{-\lambda \tau_{1}}\left(T_{11} \lambda^{2}+T_{21} \lambda+T_{31}\right),
$$

where

$$
\begin{aligned}
A_{11}= & 2 \mu_{h}+\gamma_{h}+\rho+\theta+\mu_{v}+b \beta_{v} \hat{i}_{h} \\
A_{21}= & \left(\mu_{h}+\rho\right)\left(\gamma_{h}+\mu_{h}+\theta\right)+\left(\mu_{h}+\rho\right) \mu_{v} \\
& +\left(\gamma_{h}+\mu_{h}+\theta\right) \mu_{v}+\left(\mu_{h}+\rho+\gamma_{h}+\mu_{h}+\theta\right) b \beta_{v} \hat{i}_{h} \\
A_{31}= & \left(\mu_{h}+\rho\right)\left(\gamma_{h}+\mu_{h}+\theta\right) \mu_{v} \\
& +\left(\mu_{h}+\rho\right)\left(\gamma_{h}+\mu_{h}+\theta\right) b \beta_{v} \hat{i}_{h} \\
T_{11}= & -m b \beta_{h} \hat{i}_{v} e^{-\mu_{h} \tau_{1}}, \\
T_{21}= & \left(\gamma_{h}+\mu_{h}+\theta\right) \mu_{v} \\
& -\left(\gamma_{h}+\mu_{h}+\rho+\mu_{v}+b \beta_{v} \hat{i}_{h}\right) m b \beta_{h} \widehat{i}_{v} e^{-\mu_{h} \tau_{1}} \\
T_{31}= & \left(\mu_{h}+\rho\right)\left(\gamma_{h}+\mu_{h}+\theta\right) \mu_{v} \\
& -\left(\gamma_{h}+\mu_{h}+\rho\right)\left(\mu_{v}+b \beta_{v} \hat{i}_{h}\right) m b \beta_{h} \widehat{i}_{v} e^{-\mu_{h} \tau_{1}} .
\end{aligned}
$$

By the implicit function theorem and the continuity of the left-hand side function of (18), all roots of (22) have negative real parts for small $\tau_{1}$. Notice that the condition $R_{0}>1$ is equivalent to

$$
\tau_{1}<\tau_{1}^{*}=\frac{1}{\mu_{h}} \ln \frac{m b^{2} \beta_{h} \beta_{v}}{\mu_{v}\left(\gamma_{h}+\mu_{h}+\theta\right)} .
$$

Furthermore, we claim that (22) does not have any nonnegative real roots for any $\tau_{1}>0$. Rewrite (22) by moving the positive terms from the right-hand side to the left-hand side. The rewritten (22) takes the form

$$
\lambda^{3}+A_{11} \lambda^{2}+\widetilde{A}_{21} \lambda+\widetilde{A}_{31}=e^{-\lambda \tau_{1}}\left(T_{11} \lambda^{2}+\widetilde{T}_{21} \lambda+\widetilde{T}_{31}\right),
$$

where $\widetilde{A}_{21}=A_{21}-\left(\gamma_{h}+\mu_{h}+\theta\right) \mu_{v} e^{-\lambda \tau_{1}}, \widetilde{A}_{31}=A_{31}-\left(\mu_{h}+\rho\right)$ $\left(\gamma_{h}+\mu_{h}+\theta\right) \mu_{v} e^{-\lambda \tau_{1}}$. It is easy to see $\widetilde{A}_{21}>0$ and $\widetilde{A}_{31}>0$ for all $\lambda \geq 0$ and $\tau_{1} \in\left(0, \tau_{1}^{*}\right)$. Consequently, the left-hand side in (25) is positive for all $\lambda \geq 0$ while the right-hand side is negative for all $\lambda \geq 0$ and the two cannot be equal for any $\lambda \geq 0$. Therefore, $(22)$ does not have any nonnegative real roots for any $\tau_{1} \in\left(0, \tau_{1}^{*}\right)$. Now we want to show that all roots of $(22)$ have negative real parts for all $\tau_{1} \in\left(0, \tau_{1}^{*}\right)$. To do so, we show that (22) does not have any purely imaginary roots for all $\tau_{1} \in$ $\left(0, \tau_{1}^{*}\right)$.

We assume that $\lambda=i \omega$ with $\omega>0$ being a root of (22). Then $\omega$ must satisfy the following system:

$$
\begin{gathered}
A_{21} \omega-\omega^{3}=T_{21} \omega \cos \left(\omega \tau_{1}\right)-\left(T_{31}-T_{11} \omega^{2}\right) \sin \left(\omega \tau_{1}\right), \\
A_{31}-A_{11} \omega^{2}=\left(T_{31}-T_{11} \omega^{2}\right) \cos \left(\omega \tau_{1}\right)+T_{21} \omega \sin \left(\omega \tau_{1}\right) .
\end{gathered}
$$

Thus, $\omega$ must be a positive root of

$$
\omega^{6}+B_{1} \omega^{4}+B_{2} \omega^{2}+B_{3}=0,
$$


where

$$
\begin{gathered}
B_{1}=A_{11}^{2}-2 A_{21}-T_{11}^{2}, \\
B_{2}=A_{21}-2 A_{11} A_{31}+2 T_{11} T_{31}-T_{21}^{2}, \\
B_{3}=A_{31}^{2}-T_{31}^{2} .
\end{gathered}
$$

Let $z=\omega^{2}$, then (27) becomes

$$
z^{3}+B_{1} z^{2}+B_{2} z+B_{3}=0
$$

Clearly, If $B_{1} \geq 0, B_{2} \geq 0$ and $B_{3} \geq 0$, then (29) has no positive real roots. Therefore, (22) does not have any purely imaginary roots for all $\tau_{1} \in\left(0, \tau_{1}^{*}\right)$ so that all roots of the characteristic equation (22) have negative real parts and the endemic equilibrium $P^{*}$ of (3) is stable.

Case 3. When $\tau_{2}>0, \tau_{1}=0$, the characteristic equation (18) becomes

$$
\lambda^{3}+A_{12} \lambda^{2}+A_{22} \lambda+A_{32}=e^{-\lambda \tau_{2}}\left(T_{12} \lambda^{2}+T_{22} \lambda+T_{32}\right),
$$

where

$$
\begin{aligned}
A_{12}= & 2 \mu_{h}+\gamma_{h}+\rho+\theta+\mu_{v}+m b \beta_{h} \hat{i}_{v}, \\
A_{22}= & \left(\mu_{h}+\rho\right)\left(\gamma_{h}+\mu_{h}+\theta\right)+\left(\mu_{h}+\rho\right) \mu_{v} \\
& +\left(\gamma_{h}+\mu_{h}+\theta\right) \mu_{v}+\left(\mu_{h}+\rho+\gamma_{h}+\mu_{v}\right) m b \beta_{h} \hat{i}_{v}, \\
A_{32}= & \left(\mu_{h}+\rho\right)\left(\gamma_{h}+\mu_{h}+\theta\right) \mu_{v}+\mu_{v}\left(\gamma_{h}+\mu_{h}+\rho\right) m b \beta_{h} \hat{i}_{v} \\
T_{12}= & -b \beta_{v} \hat{i}_{h} e^{-\mu_{v} \tau_{2}}, \\
T_{22}= & \left(\gamma_{h}+\mu_{h}+\theta\right) \mu_{v} \\
& -\left(2 \mu_{h}+\gamma_{h}+\rho+\theta+m b \beta_{h} \hat{i}_{v}\right) b \beta_{v} \hat{i}_{h} e^{-\mu_{v} \tau_{2}}, \\
T_{32}= & \left(\mu_{h}+\rho\right)\left(\gamma_{h}+\mu_{h}+\theta\right) \mu_{v} \\
& -\left(\gamma_{h}+\mu_{h}+\rho\right) m b^{2} \beta_{h} \beta_{v} \widehat{i}_{h} \widehat{i}_{v} e^{-\mu_{v} \tau_{2}} \\
& -\left(\mu_{h}+\rho\right)\left(\gamma_{h}+\mu_{h}+\theta\right) b \beta_{v} \hat{i}_{h} e^{-\mu_{v} \tau_{2}} .
\end{aligned}
$$

The condition $R_{0}>1$ is equivalent to

$$
\tau_{2}<\tau_{2}^{*}=\frac{1}{\mu_{v}} \ln \frac{m b^{2} \beta_{h} \beta_{v}}{\mu_{v}\left(\gamma_{h}+\mu_{h}+\theta\right)} .
$$

Using a similar argument as in Case 2, we know that all roots of (30) have negative real parts for $\tau_{2} \in\left(0, \tau_{2}^{*}\right)$ when $C_{1} \geq$ $0, C_{2} \geq 0$, and $C_{3} \geq 0$, where

$$
\begin{gathered}
C_{1}=A_{12}^{2}-2 A_{22}-T_{12}^{2}, \\
C_{2}=A_{22}-2 A_{12} A_{32}+2 T_{12} T_{32}-T_{22}^{2}, \\
C_{3}=A_{32}^{2}-T_{32}^{2} .
\end{gathered}
$$

Case 4. When $\tau_{2}>0, \tau_{1}>0$, the condition $R_{0}>1$ is equivalent to

$$
\tau_{2}<\tau_{2}^{*}\left(\tau_{1}\right)=\frac{1}{\mu_{v}} \ln \frac{m b^{2} \beta_{h} \beta_{v} e^{-\mu_{h} \tau_{1}}}{\mu_{v}\left(\gamma_{h}+\mu_{h}+\theta\right)} .
$$

From Cases 1, and 2 the roots of (18) only have negative real parts for $\tau_{1} \in\left[0, \tau_{1}^{*}\right)$ and $\tau_{2}=0$ under the condition of $B_{i} \geq 0, i=1,2,3$. By the implicit function theorem and the continuity of the left-hand side function of (18), there is a $\bar{\tau}_{2}\left(\tau_{1}\right)$ satisfying $0<\bar{\tau}_{2}\left(\tau_{1}\right) \leq \tau_{2}^{*}\left(\tau_{1}\right)$, such that all roots of (18) have negative real parts for $0<\tau_{2}<\bar{\tau}_{2}\left(\tau_{1}\right)$. We show that $\bar{\tau}_{2}\left(\tau_{1}\right)=\tau_{2}^{*}\left(\tau_{1}\right)$ when $B_{i} \geq 0$ and $C_{i} \geq 0, i=1,2,3$. Suppose $\bar{\tau}_{2}\left(\tau_{1}\right)<\tau_{2}^{*}\left(\tau_{1}\right)$ for $\tau_{1} \in\left[0, \tau_{1}^{*}\right)$, then there must be a $\widetilde{\tau}_{2}\left(\tau_{1}\right), \bar{\tau}_{2}\left(\tau_{1}\right)<\widetilde{\tau}_{2}\left(\tau_{1}\right)<\tau_{2}^{*}\left(\tau_{1}\right)$, such that one root of (18) has nonnegative real part for $\tau_{2}=\tilde{\tau}_{2}\left(\tau_{1}\right)$. As a result of the continuity of $\tau_{2}$ in $\tau_{1}$, we have $\tilde{\tau}_{2}(0)<\tau_{2}^{*}(0)=\tau_{2}^{*}$. However, from the argument in Case 3, we know that all the roots of (18) have negative real parts for $\tau_{1}=0$ and $\tau_{2} \in\left(0, \tau_{2}^{*}\right)$. Contradict. Thus, $\bar{\tau}_{2}\left(\tau_{1}\right)=\tau_{2}^{*}\left(\tau_{1}\right)$ which implies that the endemic equilibrium $P^{*}$ is stable when $\tau_{1} \in\left[0, \tau_{1}^{*}\right)$, $\tau_{2} \in\left[0, \tau_{2}^{*}\left(\tau_{1}\right)\right), B_{i} \geq 0$, and $C_{i} \geq 0, i=1,2,3$. The above analysis can be summarized into the following theorem.

Theorem 6. If $R_{0}>1, B_{i} \geq 0$ and $C_{i} \geq 0, i=1,2,3$, the unique endemic equilibrium $P^{*}$ of system (3) is stable.

\section{Discussions}

In this paper, in order to study the impact of the incubation periods in human and mosquitoes, we formulate a model with two delays for the transmission dynamics of malaria.

Existence of equilibria is obtained under different conditions and their stabilities are analyzed too. We have also identified the basic reproduction number $R_{0}$, which gives the expected number of new infections (in mosquitoes or humans) from one infectious individual (human or mosquito) over the duration of the infectious period, given that all other members of the population are susceptible, in terms of the model parameters. Especially, we have proved mathematically backward bifurcation may occur for $R_{0}<1$ which implies that bringing the basic reproduction number below 1 is not enough to eradicate malaria.

The parameters $\tau_{1}$ and $\tau_{2}$ are the incubation periods in human and mosquitoes, which are temperature dependent. With the increasing of temperature in a range, they become shorter. Since the basic reproduction number is a monotone decreasing function of both time delays, global warming will then exacerbate the transmission of malaria.

The reason inducing backward bifurcation in [6] is the mosquito biting rate. While, the reasons inducing the backward bifurcation in our paper are the standard incidence rate and the disease-induced death rate which is big enough. By our results, the disease-free equilibrium is globally stable when $R_{0}<1$ if the disease-induced death is omitted. However, the result about backward bifurcation in this paper has important implications for malaria control, which implies 
that bringing the basic reproduction number below 1 is not enough to eradicate malaria.

Malaria transmission can be affected by a lot of aspects. In this paper, we are trying to model the impact of temperature increasing on malaria transmission. In some extent, our results can provide a theoretical principle for allocating and using medical health resource reasonably, which can be applied in the practice of malaria prevention and control.

\section{Acknowledgments}

H. Wan is supported by NSFC (no. 11201236 and no. 11271196) and the NSF of the Jiangsu Higher Education Committee of China (no. 11KJA110001 and no. 12KJB110012). J.-a. Cui is supported by NSFC (no. 11071011) and Funding Project for Academic Human Resources Development in Institutions of Higher Learning Under the Jurisdiction of Beijing Municipality (no. PHR201107123).

\section{References}

[1] N. T. J. Bailey, "The Biomathematics of malaria," in Malaria: Principles and Practice of Malariology, Oxford University Press, London, UK, 1988.

[2] H. H. Diebner, M. Eichner, L. Molineaux, W. E. Collins, G. M. Jeffery, and K. Dietz, "Modelling the transition of asexual blood stages of Plasmodium falciparum to gametocytes," Journal of Theoretical Biology, vol. 202, no. 2, pp. 113-127, 2000.

[3] H. M. Yang, "Malaria transmission model for different levels of acquired immunity and temperature-dependent parameters (vector)," Journal of Public Health, vol. 34, no. 3, pp. 223-231, 2000.

[4] R. M. Anderson and R. M. May, Infectious Diseases of Humans: Dynamics and Control, Oxford University Press, Oxford, UK, 1991.

[5] L. J. Bruce-Chwatt, Essential Malariology, Heinemann, London, UK, 1980.

[6] N. Chitnis, J. M. Cushing, and J. M. Hyman, "Bifurcation analysis of a mathematical model for malaria transmission," SIAM Journal on Applied Mathematics, vol. 67, no. 1, pp. 24-45, 2006.

[7] Z. Hu, Z. Teng, and H. Jiang, "Stability analysis in a class of discrete SIRS epidemic models," Nonlinear Analysis. Real World Applications, vol. 13, no. 5, pp. 2017-2033, 2012.

[8] R. Ross, The Prevention of Malaria, Murry, London, UK, 1911.

[9] S. Ruan, D. Xiao, and J. C. Beier, "On the delayed RossMacdonald model for malaria transmission," Bulletin of Mathematical Biology, vol. 70, no. 4, pp. 1098-1114, 2008.

[10] Y. Takeuchi, W. Ma, and E. Beretta, "Global asymptotic properties of a delay SIR epidemic model with finite incubation times," Nonlinear Analysis. Theory, Methods \& Applications A, vol. 42, no. 6, pp. 931-947, 2000.

[11] J. Tumwiine, L. S. Luboobi, and J. Y. T. Mugisha, "Modelling the eect of treatment and mosquitoes control on malaria transmission," International Journal of Management and Systems, vol. 21, pp. 107-124, 2005.

[12] H. Wan and J.-A. Cui, "A model for the transmission of malaria," Discrete and Continuous Dynamical Systems. Series B, vol. 11, no. 2, pp. 479-496, 2009.
[13] W. Wang and S. Ruan, "Bifurcation in an epidemic model with constant removal rate of the infectives," Journal of Mathematical Analysis and Applications, vol. 291, no. 2, pp. 775-793, 2004.

[14] X. Zhou and J. Cui, "Delay induced stability switches in a viral dynamical model," Nonlinear Dynamics, vol. 63, no. 4, pp. 779792, 2011.

[15] H.-M. Wei, X.-Z. Li, and M. Martcheva, "An epidemic model of a vector-borne disease with direct transmission and time delay," Journal of Mathematical Analysis and Applications, vol. 342, no. 2, pp. 895-908, 2008.

[16] H. W. Hethcote, "The mathematics of infectious diseases," SIAM Review, vol. 42, no. 4, pp. 599-653, 2000.

[17] S. M. Garba, A. B. Gumel, and M. R. Abu Bakar, "Backward bifurcations in dengue transmission dynamics," Mathematical Biosciences, vol. 215, no. 1, pp. 11-25, 2008.

[18] R. M. Anderson and R. M. May, Population Biology of Infectious Diseases, Springer, Berlin, Germany, 1982. 


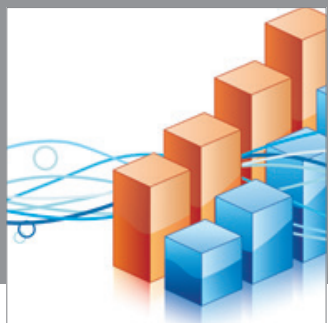

Advances in

Operations Research

mansans

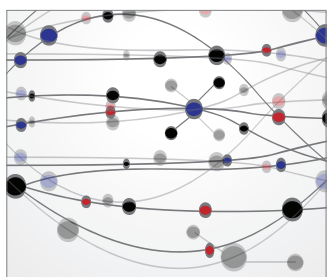

The Scientific World Journal
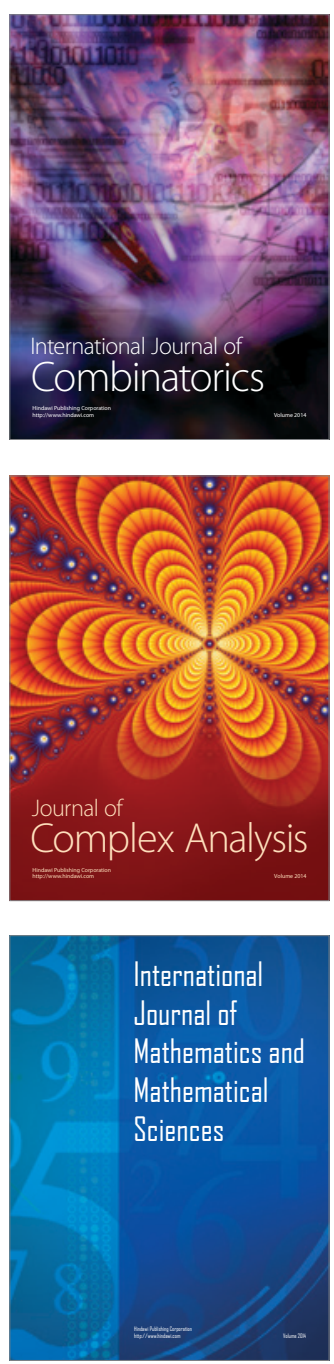
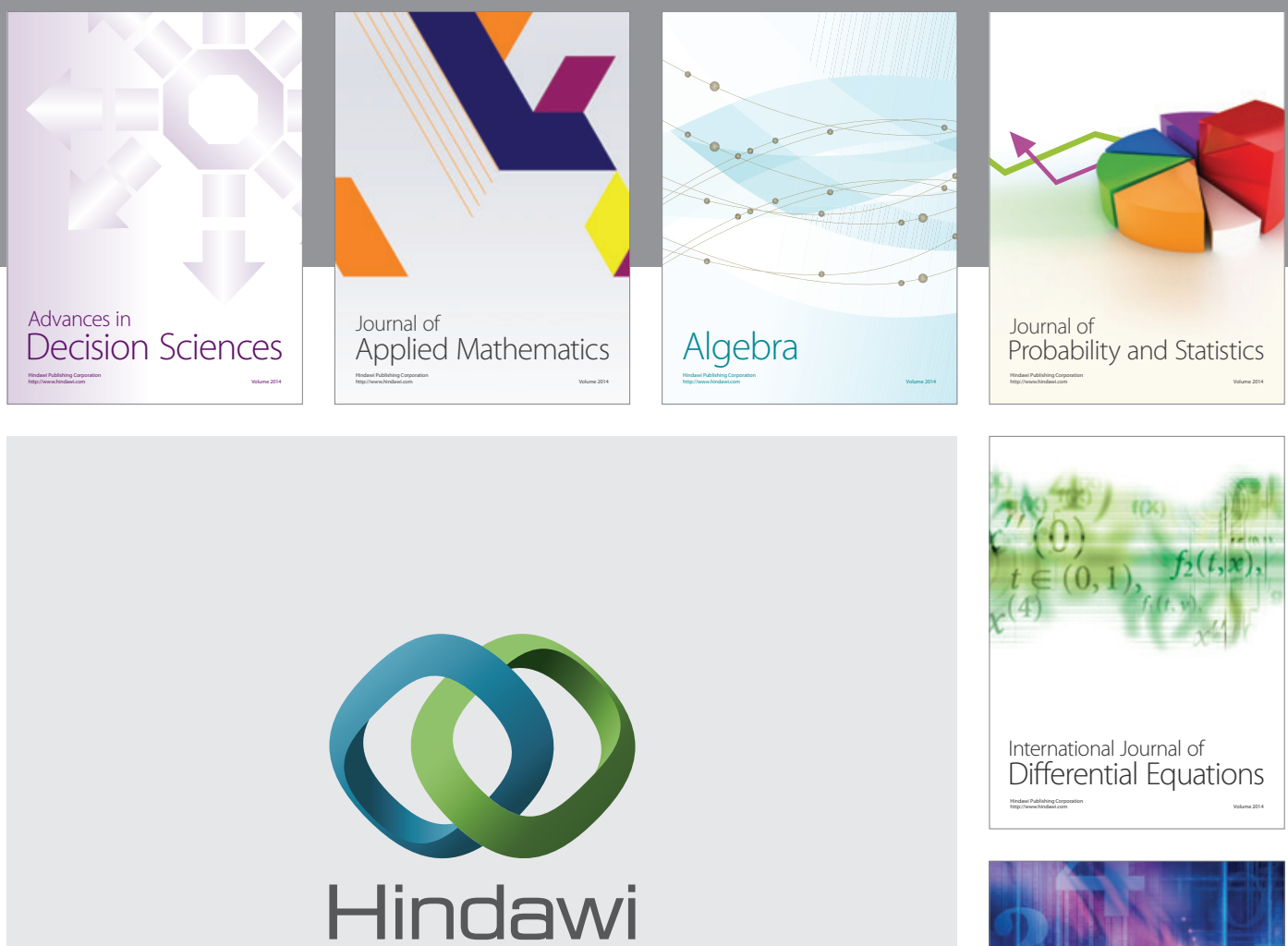

Submit your manuscripts at http://www.hindawi.com
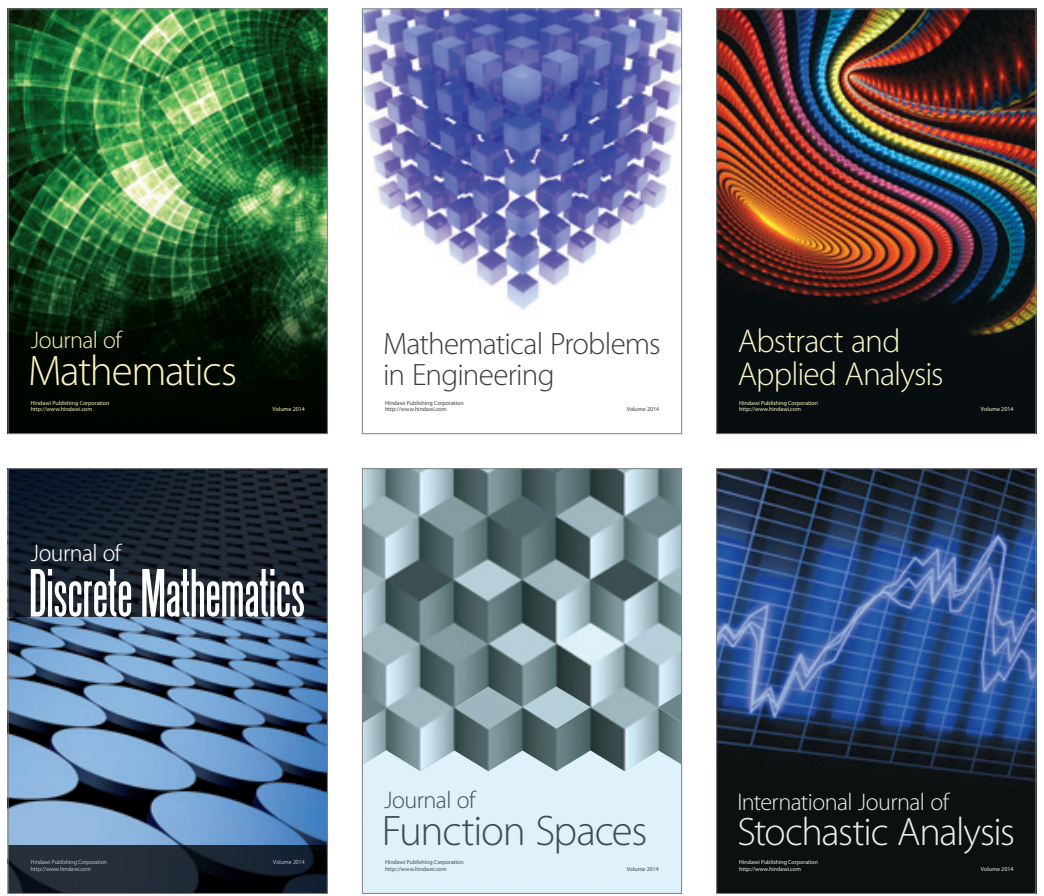

Journal of

Function Spaces

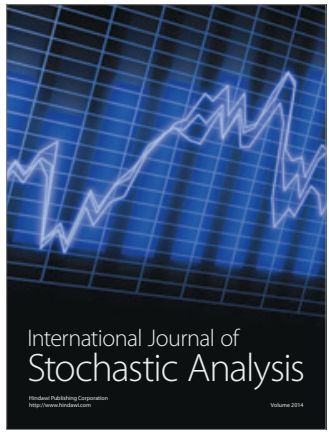

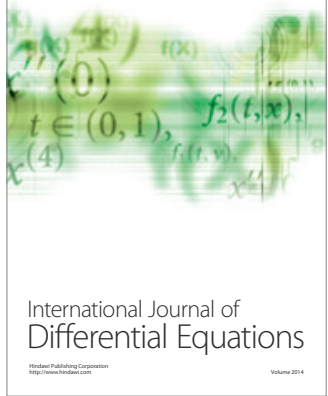
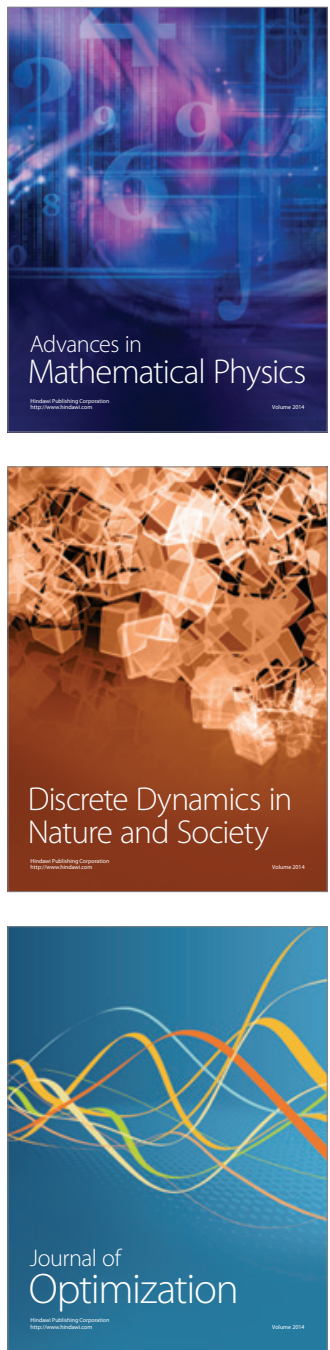